Pacific Journal of Mathematics

RIGHT SIMPLE CONGRUENCES ON A SEMIGROUP 


\title{
RIGHT SIMPLE CONGRUENCES ON A SEMIGROUP
}

\author{
FRANCIS E. MASAT
}

The purpose of this paper is to investigate necessary and sufficient conditions on an algebraic semigroup in order that it have non-trivial right simple homomorphic images. Relative to this, the relation between the structure of $S$ and the structure of its right simple homomorphs is characterized.

The main questions considered are:

(1) What characterizes a right simple (right group) congruence on a semigroup?

(2) Can the conditions found for question (1) be made minimal in order that a maximum right simple homomorph occurs?

In $\S 2$, question (1) is answered in terms of right neat subsets of a semigroup. The concepts of minimal right neat subsets and minimal right ideals are used in $\$ 3$ to obtain some sufficient conditions in order that question (2) may be answered in the affirmative. Relative to the stated sufficient conditions, a structure theorem is given. Also in $\$ 3$, left cancellative congruences are used to generate right group homomorphs of a semigroup.

The right-left duals of all results established will be taken for granted without further comment. For basic concepts, definitions, and terminology, the reader is referred to Clifford and Preston (1). Also, $S \backslash I$ denotes set difference, $|S|$ denotes the cardinality of the set $S$.

2. Right simple congruences. A semigroup $S$ is called right [left] simple if it contains no proper right [left] ideal. A group, then, is just a semigroup that is both left and right simple, [1, p. 39]. A semigroup $S$ is called a right group if it is right simple and left cancellative. This means that for any $a$ and $b$ in $S$ there exists a unique $x$ in $S$ such that $a x=b$.

A semigroup $S$ is said to be regular if for each $a$ in $S, a$ is also in $a S a$. When $S$ is regular, the set of idempotents of $S$ is nonempty and will be denoted by $E_{S}$. If there is no danger of ambiguity, $E$ will be used instead of $E_{S}$.

The following results are stated for later application.

LEMMA 2.1. [1, Lemma 1.26, p. 37]. Every idempotent element of a right simple semigroup $S$ is a left identity element of $S$. 
LEMMA 2.2 [1]. The following assertions concerning a semigroup $S$ are equivalent:

(1) $S$ is a right group.

(2) $S$ is right simple, and contains an idempotent.

(3) $S$ is a direct product $G \times E$ of a group $G$ and a right zero semigroup E.

(4) $S$ is a union of isomorphic disjoint groups such that the set of identity elements of the groups is a right zero subsemigroup of $S$.

(5) $S$ is regular and left cancellative.

REMARK 2.3. When a congruence $\rho$ is such that $S / \rho$ is the maximal homomorphic image of $S$ of type $C$, as in [2, p. 275] and [2, Theorem 11. 25(A), p. 276], then $\rho$ will be called the minimum congruence on $S$ of type $C$ and $S / \rho$ will be called the maximum homomorphic image of $S$ of type $C$.

In other words, $S / \rho$ is the maximum $C$-image if and only if $\rho$ is of type $C$ and $\rho \subseteq \sigma$ for each congruence $\sigma$ which is of type $C$. Moreover, the phrases "right group congruence" and "group congruence" will be denoted by $R G C$ and $G C$ respectively. When such a congruence is minimum, it will be denoted by $M R G C$ and $M G C$ respectively.

The preceding proposition, (2.3), would seem to indicate that the minimum right simple [right group, group] congruence on a semigroup $S$ could be found by considering the intersection of all the right simple [right group, group] congruences on $S$. However, the intersection of the right simple congruences on a semigroup $S$ need not be a right simple congruence on $S$.

EXAMPLE 2.4. Let $S$ be the additive semigroup of positive integers. The group images of $S$ are exactly all the finite multiplicative cyclic groups and there is no maximal group among these. The intersection of the induced congruences on $S$ is the identity congruence $\iota$, but $\iota$ is not a right simple congruence since $S$ is not right simple. Hence $\iota$ is neither a right group congruence nor a group congruence.

PROPOSITION. Every homomorphic image of a right simple semigroup is right simple.

Alternately, a semigroup is right simple if and only if its minimum right simple congruence is the identity congruence.

A subset $X$ of a semigroup $S$ is said to be a right [left] neat subset of $S$ if for each $a$ in $S$ there exists $s$ in $S$ such that $a s \in X[s a \in X]$. Right [left] neat 
subsets may also be viewed as those subsets of $S$ which meet (have nonempty intersection with) each right [left] ideal of the form $a S[S a]$ where $a$ is in $S$. A set $U$ is a cross-section of a collection $\left\{X_{a}: a \in A\right\}$ of sets if and only if $\left|U \cap X_{a}\right|=1$ for all $a$ in $A$.

If a semigroup $S$ has proper right ideals, define the subset $U$ of $S$ to be any cross-section of the set of all (proper) right ideals of $S$. The set $U$ meets each principal right ideal of $S$ and hence $U$ is a proper right neat subset of $S$. The converse is false since each singleton subset of a right simple semigroup is right neat.

The next theorem shows the close relationship between the right neat subsets of a semigroup $S$ and the right simple congruences on $S$.

THEOREM. A homomorph $S \theta$ of a semigroup $S$ is right simple if and only if each congruence class of $S$ modulo $\theta \circ \theta^{-1}$ is right neat.

The following theorem characterizes those right simple congruences on a semigroup which are right group congruences.

THEOREM 2.5. A necessary and sufficient condition that a right simple. congruence on a semigroup $S$ be a right group congruence is that some congruence class contain a subsemigroup of $S$.

The next theorem generalizes a result due to Fontaine $[4, \mathrm{Thm} .12, \mathrm{p}$. 784].

THEOREM 2.6. Suppose a semigroup $S$ has a right neat subset $K$. Let $\rho$ be a congruence on $S$ such that for each $b$ in $S$ and for each $k$ in $K$, there exists an $x$ in $S$ such that $(k x) \rho b$. Then $S / \rho$ is a right simple semigroup.

Proof. For $a$ in $S$, there exists $y$ in $S$ such that $a y \in K$. Hence for $b$ in

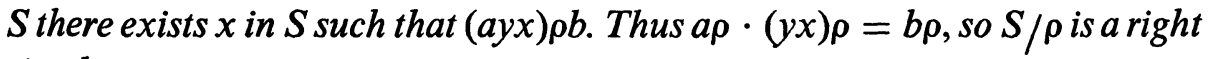
simple semigroup.

COROllary [4, Thm.12, p. 784]. Let $\rho$ be a congruence on a semigroup $S$ containing a right neat subset $K$. If $(k b) \rho b$ for each $b$ in $S$ and for each $k$ in $K$, then $\rho$ is a right group congruence on $S$.

The next result shows what happens to the right ideals of a semigroup under a right simple homomorphism.

THEOREM 2.7. If $\theta$ is a homomorphism of a semigroup $S$ onto a right simple semigroup $S^{\prime}$, then $\theta$ maps every right ideal of $S$ onto $S^{\prime}$. 
Proof. If $I$ is a right ideal of $S$, then $(I) \theta$ is a right ideal of $S^{\prime}$, and therefore $(I) \theta=S^{\prime}$.

COROLlary 2.8. If a semigroup $S$ has a left zero, then the minimum right simple congruence on $S$ is the universal congruence $\omega$.

Semigroups with a right zero are considered in \$3. A second corollary is the following theorem of Lefebrve [6, Lemma 1, p. 2277].

COROLlaRY 2.9. If $\theta$ is a homomorphism of a semigroup $S$ onto a group $S^{*}$, then $\theta$ maps every ideal of $S$ onto $S^{*}$.

The following result is a partial converse for Theorem 2.7.

THEOREM. If $\theta$ is a homomorphism of a right ideal $R$ of a semigroup $S$ onto a right group $S^{*}$, and if $R$ has a right identity, then $\theta$ can be extended to a homomorphism of $S$ onto $S^{*}$.

Proof. Define a mapping $g$ from $S$ to $S^{*}$ by $(s) g=(i s) \theta$, where $i$ is the right identity of $R$. It is evident that $g$ is well-defined, maps $S$ homomorphically onto $S^{*}$, and equals $\theta$ when restricted to $R$.

3. Minimum right simple congruences. If $X$ is a right [left] neat subset of a semigroup $S$ and if $x$ is in $X$, then the set of all $s$ in $S$ such that $x s$ $\in X[s x \in X]$ is denoted by $x^{[-1]} X\left[X x^{[-1]}\right]$. In the following development the set $x S \cap X[S x \cap X]$ will be denoted by $x\left(x^{[-1]} X\right)\left[\left(X x^{[-1]}\right) x\right]$.

The following theorem of Dubreil [3, Thm. 1, p. 34] characterizes those right neat subsets of a semigroup which are minimal.

THEOREM 3.1. In order that a right neat subset $K$ of a semigroup $S$ be minimal, it is necessary and sufficient that for each $k$ in $K, k\left(k^{[-1]} K\right)=\{k\}$.

Additionally we need the following results from Lefebvre concerning semigroups possessing MRN (minimal right neat) subsets and MRI's (minimal right ideals).

THEOREM 3.2. Every minimal right neat subset $K$ of $S$ has the following properties:

(i) For each $k \in K$, there exists $x \in S$ such that $k x=k$.

(ii) For each $a \in S$ and for each $k \in K$, there exists $x \in S$ such that $k a x=k$. 
(iii) For each $k_{1}, k_{2} \in K$ and for each $x_{1}, x_{2} \in S$, if $k_{1} x_{1}=k_{2} x_{2}$ then $k_{1}=k_{2}$.

(iv) For each $a \in S, K a$ is a minimal right neat subset of $S$.

Proof. [5, Properties 1, 2, 3 and Theorem 2, p. 394].

THEOREM 3.3. Let $S$ be a semigroup with minimal right neat subsets. Then:
(i) Every right neat subset of $S$ contains a minimal right neat subset of $S$.
(ii) S contains a minimal right ideal.
(iii) Any two minimal right neat subsets of $S$ correspond bijectively.

Proof. [5, Thm. 4, Cor. 2, and Thm. 3, pp. 394-395].

THEOREM 3.4. If a semigroup $S$ contains a minimal right ideal, it contains a minimal right neat subset. Moreover, the union of the minimal right neat subsets of $S$ coincides with the union of the minimal right ideals of $S$.

Proof. [5, Theorem 7, p. 395]. It should be noted that the MRN subsets of $S$ are formed by taking cross-sections of the MRI's of $S$. Denote the union of the $M R N$ subsets of $S$ by $N$, and the union of the MRI's of $S$ by $R$. That $R=N$ follows from [5, Corollary 3, p. 395].

The next result shows the cardinality and structure relationship between minimal right ideals of $S$ and minimal right neat subsets of $S$.

COROLlaRY 3.5. Let $S$ have a minimal right ideal. Then:

(i) Every minimal right neat subset of $S$ is a cross-section of the set of minimal right ideals of $S$.

(ii) The number of elements in any minimal right neat subset of $S$ equals the number of minimal right ideals of $S$.

(iii) The following are equivalent:

(A) The minimal right neat subsets of $S$ are mutually disjoint.

(B) The minimal right neat subsets of $S$ are singletons.

(C) S contains exactly one minimal right ideal.

Proof. Denote the set of $M R I$ 's of $S$ by $\left\{R_{a}: a \in A\right\}$ for an index set 
$A$, and denote the set of $M R N$ subsets of $S$ by $\left\{K_{i}: i \in I\right\}$ for an index set I.

(i) By Theorem 3.4, any cross-section of $\left\{R_{a}: a \in A\right\}$ is a $M R N$ subset of $S$. Conversely, for $i$ in $I, K_{i} \cap R_{a} \neq \square$ for each $a$ in $A$. If $a$ in $A$ and $h, k \in K_{i} \cap R_{a}$, then $k R_{a}=h R_{a}=R_{a}$. It follows from Theorem 3.2 (iii) that $k=h$.

(ii) By Theorem 3.3 (iii), the number of elements in any $K_{i}$ is independent of $i$ in $I$, and hence, by (i), is equal to $|A|$.

(iii) Proceeding indirectly, assume some (and hence every) $K_{i}$ contains more than one element, and consider the set $K$ defined by $K=$ $\left(K_{i} \cap R_{a}\right) \cup\left(K_{j} \backslash R_{a}\right)$, for a fixed $i, j$ in $I, a$ in $A$. By part (i), $K$ is a MRN subset of $S$, but $K \cap K_{i} \neq \square$. Thus (A) implies (B).

If the $K_{i}$ 's are all singletons, then by (ii), (B) implies (C).

If $|A|=1$, then by (i), a cross-section of the MRI is precisely a MRN singleton subset and therefore (C) implies (A).

The following theorem relates the MRN subsets of a semigroup $S$ to the right simple congruences on $S$.

THEOREM 3.6. Let $S$ be a semigroup with minimal right ideals. Then:

(i) S is a disjoint union of right neat subsets.

(ii) The decomposition of $S$ in (i) induces a right congruence $\rho$ on $S$.

(iii) $\rho$ can be characterized as follows. For some minimal right neat subset $K$ of $S$ and for a fixed $x$ in $K$,

$$
\rho=\{(s, t) \in S \times S: x s=x t\} .
$$

(Accordingly, we write $\rho=\rho_{x}$.)

(iv) $\rho_{x}$ is right simple in that for each $a, b$ in $S$ there exists $u$ in $S$ such that $(a u) \rho_{x}=b \rho_{x}$.

Proof. (i) Let $x$ be fixed in some MRN subset $K$. For each $s$ in $S$, $x s$ in $K s$ implies that $s \in x^{[-1]} K s$. For convenience, denote $x^{[-1]} K s$ by [s]. Thus

$$
S=\cup\{[s]: s \in S\}
$$

If $[s] \cap[r] \neq \square$ for some $r$ and $s$ in $S$, then there exists $b$ in $S$ such that $x b \in K s \cap K r$. Hence there exists $h, k$ in $K$ such that $x b=h s$ and $x b=k r$. But then by Theorem 3.2 (iii), $h=x=k$ and so $x r=x b=x$ s. Hence if $c$ 
$\in[s]$ then $x c=x s=x r$, so $c \in[r]$. Therefore $[s] \subseteq[r]$, and similarly $[r] \subseteq$ $[s]$. Hence $[s]=[r]$ and the decomposition (3.7) is a partition of $S$.

Let $Y$ denote a set of representatives for $\{[s]: s \in S\}$. Thus

$$
S=U\{[y]: y \in Y\}
$$

and this union is disjoint. For $a$ in $S$ and $y$ in $Y$, the set $K y$ is right neat and so there exists $z$ in $S$ such that $(x a) z \in K y$, i.e., $a z \in[y]$ and therefore [y] is right neat.

(ii) Let $\rho_{x}$ denote the equivalence relation induced on $S$ by the partition (3.8). If $(s, t) \in \rho_{x}$ and $c \in S$, then there exists $y$ in $Y$ such that $s$, $t \in[y]$ and hence $x s=x t=x y$. Thus $x s c=x t c=x y c$, so $s c, t c \in[y c]$. Assuming $[y c]$ meets $\left[y^{\prime}\right]$ for $y^{\prime} \in Y$, implies by part (i) that $\rho_{x}$ is right compatible.

(iii) If $(s, t) \in \rho_{x}$ then $x s=x t=x y$ for some $y$ in $Y$. Conversely, suppose $x s=x t$. For some $y$ in $Y, s \in[y]$ and thus $x s=x t=x y$, i.e., $(s, t)$ $\in \rho_{x}$.

(iv) Since $x \in K$, a MRN subset of $S, x$ lies in a MRI $R$ of $S$; thus $x S$ $=R$. For any $a, b$ in $S$, there exists $u$ in $S$ such that $x a u=x b$ since $x S=$ $x a S=R$. Hence by the definition of $\rho_{x}, a u \rho_{x} b$.

The following definitions will be used frequently. Let $S$ be a semigroup and let $X$ be a subset of $S$.

$$
\begin{aligned}
& \text { For } x \text { in } S \text {, let } \rho_{x}=\{(a, b) \in S \times S: x a=x b\} \\
& \rho_{X}=\{(a, b) \in S \times S: x a=x b \text { for all } x \text { in } X\}
\end{aligned}
$$

Remark 3.11. Note that $\rho_{X}=\cap\left\{\rho_{x}: x \in X\right\}$, and that for each $x$ in $X, \rho_{x}$ is a right congruence on $S$. Hence, $\rho_{X}$ is also a right congruence on $S$.

The above definitions are motivated by the observation that a right simple congruence is a right group congruence if and only if it is left cancellative. The following remark easily generalizes to the left [right] cancellative case.

REMARK 3.12. [2, Lemma 9.49, p. 164]. Let $\left\{\sigma_{i}: i \in I\right\}$ be the family of all cancellative congruences on a semigroup $S$. Then $\sigma=\cap\left\{\sigma_{i}: i \in I\right\}$ is a cancellative congruence on $S$.

Since $\omega$ is a cancellative congruence on any semigroup $S$, the foregoing remark shows that there exists a (unique) minimum left cancellative congruence on $S$.

Define $\lambda$ by 


$$
\lambda=\{(a, b) \in S \times S: x a=x b \text { for some } x \text { in } S\} .
$$

It is evident that $\lambda$ is a reflexive, symmetric, and left cancellative relation on $S$. If $\sigma$ is any left cancellative relation on $S$ and $(a, b) \in \lambda$, then $x a=x b$ for some $x$ in $S$, so $(x a, x b) \in \sigma$. By the left cancellativity of $\sigma,(a, b) \in \sigma$; therefore $\lambda \subseteq \sigma$. Thus if $\lambda$ is a congruence on $S$, then it is the minimum left cancellative congruence on $S$. A major difficulty with $\lambda$ is in its being transitive for an arbitrary semigroup $S$.

In the following development, the approach used to overcome the transitivity problem of $\lambda$ is to fix the element $x$ in the definition of $\lambda$. Thus for a fixed $x$ in $S, \lambda=\rho_{x}$ Relabeling $\lambda$ as $\rho_{x}, \rho_{x}$ (and hence $\rho_{X}$ ) becomes a right congruence on $S$. Moreover, $\rho_{X} \subseteq \rho_{x} \subseteq \lambda$ for all $x$ in $X$. It remains then to find conditions on $S$ such that $\rho_{x}$ is left compatible and right simple. A result in this direction is the following theorem.

THEOREM 3.14. Let $A$ be a subset of the semigroup $S$ and let $\sigma$ be $a$ congruence on $S$. Define $\sigma_{A}$ by

$$
\sigma_{A}=\cap\left\{\sigma_{x}: x \in A\right\}
$$

where, for each $x$ in $A$

$$
\sigma_{x}=\{(a, b) \in S \times S:(x a, x b) \in \sigma\} .
$$

(1) If $A$ is a right ideal of $S$, then $\sigma_{A}$ is a congruence on $S$.

(2) If $\sigma_{A}$ is left cancellative, then $\sigma_{A}=\sigma_{x}$ for each $x$ in $A$.

(3) If $A$ is a minimal right ideal of $S$, then $\sigma_{A}$ is left cancellative if and only if it is a right group congruence on $S$.

Proof. (1) The relation $\sigma_{x}$ is a right congruence on $S$ for each $x$ in $A$. Thus it suffices to show that $\sigma_{A}$ is left compatible since the intersection of right congruences is a right congruence. If $(a, b) \in \sigma_{A}$, then $(a, b) \in \sigma_{x}$ for all $x$ in $A$. If $c \in S$, then $x c \in A S \subseteq A$, so that $a \sigma_{x c} b$, that is, $x c a \sigma x c b$. But then by the definition of $\sigma_{x}, c a \sigma_{x} c b$, i.e., $(c a, c b) \in \sigma_{x}$ for each $x$ in $A$. Hence $(c a, c b) \in \sigma_{A}$ and $\sigma_{A}$ is a congruence on $S$.

(2) Suppose $\sigma_{A}$ is left cancellative. Clearly $\sigma_{A} \subseteq \sigma_{x}$ for all $x$ in $A$. Conversely let $(a, b) \in \sigma_{x}$ and $y \in A$. Since $x a \sigma x b$ and $\sigma$ is a congruence, then $(y x a, y x b) \in \sigma$ and therefore $(x a, x b) \in \sigma_{y}$ for every $y$ in $A$, i.e., ( $x a$, $x b) \in \sigma_{A}$. Since $\sigma_{A}$ is left cancellative, then $(a, b) \in \sigma_{A}$. Thus for each $x$ in $A, \sigma_{x} \subseteq \sigma_{A} \subseteq \sigma_{x}$, i.e., $\sigma_{A}=\sigma_{x}$.

(3) If $A$ is a $M R I$ of $S$ then $x A=A$ for each $x$ in $A$. Suppose $\sigma_{A}$ is left cancellative. If $a, b \in S$ then for any $x$ in $A, x a$ and $x b$ are in $A$ and therefore there exists $y$ in $A$ such that $x a y=x b$, that is, $a y \sigma_{x} b$. By (2) above, $a y \sigma_{A} b$. This shows that $S / \sigma_{A}$ is a right simple semigroup. 
If $x$ is in $A$, then there exists $y$ in $A$ such that $x y=x$. Therefore $x y^{2}=$ $x y$ which implies that $y^{2} \sigma_{x} y$ and hence that $y^{2} \sigma_{A} Y$. Thus, $y \sigma_{A}$ is an idempotent in $S / \sigma_{A}$ and therefore by Lemma 2.2 (2), $\sigma_{A}$ is a RGC.

Conversely, if $\sigma_{A}$ is a RGC on $S$ then, by definition, it is a left cancellative congruence on $S$.

Remark. The converse of Theorem 3.14 (1) is false. Let $S$ be the additive semigroup of positive integers, and let $\sigma=\iota$ and $A=\{1\}$. Then for $a, b \in S,(a, b) \in \sigma_{A}$ if and only if $(a, b) \in \sigma_{1}$, that is, if and only if $1+a=$ $1+b$. But this last equation is true if and only if $a=b$ and hence $\sigma_{A}=\sigma_{1}$ $=\sigma=\iota$. So $\sigma_{A}$ is a congruence but $A$ is not a right ideal of $S$.

The converse of Theorem 3.14 (2) is also false. Consider for example, the multiplicative semigroup of the integers modulo four, i.e., $S=\left\{0^{\prime}, 1^{\prime}\right.$, $\left.2^{\prime}, 3^{\prime}\right\}$. Choose the subset $\left\{2^{\prime}\right\}$ of $S$ to be $A$ and set $\sigma=\iota$. Then $\sigma_{A}=\sigma_{2^{\prime}}=$ $\iota \cup\{(0,2),(2,0),(1,3),(3,1)\}$. Now $\left(0^{\prime} \cdot 2^{\prime}, 0^{\prime} \cdot 3^{\prime}\right) \in \sigma_{A}$ but $\left(2^{\prime}, 3^{\prime}\right) \notin$ $\sigma_{A}$, i.e., $\sigma_{A}$ is not left cancellative.

The following special case of Theorem 3.14 is worth noting.

COROLLARY 3.15. Let $A$ be a minimal right ideal of a semigroup $S$, let $\sigma$ be the identity relation on $S$ and define $\rho_{A}$ by equation (3.10). Then: $\sigma_{A}=$ $\rho_{A}$. Moreover, $\rho_{A}$ is left cancellative if and only if $\rho_{A}$ is the minimum right group congruence on $S$.

Proof. Certainly $\sigma_{A}$ is equal to $\rho_{A}$. Now suppose $\rho_{A}$ is left cancellative. Then by part (3) of Theorem 3.14, $\rho_{A}$ is a RGC on $S$.

If $\sigma$ is RGC on $S$, then by Theorem 2.7, $\sigma$ maps $A$ onto $S / \sigma$. Hence there exists $y$ in $A$ such that $y \sigma \in E_{S / \sigma}$. For $a, b \in S$ such that $a \rho_{A} b, x a=x b$ for all $x$ in $A$. In particular then, $y a=y b$. Thus under $\sigma, y a \sigma=y b \sigma$. Since $y \sigma$ is a left identity for $S / \sigma, a \sigma=b \sigma$, that is, $\rho_{A} \subseteq \sigma$. Hence $\rho_{A}$ is the MRGC on $S$.

The converse is evident.

It is now logical to consider necessary and sufficient conditions on $S$ such that $\rho_{x}$ of equation (3.9) is a congruence. The next theorem characterizes those $\rho_{x}$ which are left compatible.

THEOREM 3.16. Let $S$ be a semigroup containing minimal right ideals, let $K$ be a minimal right neat subset of $S$ and let $x \in K$. Let $Y$ denote a set of representatives of the set of disjoint right neat subsets of $S$. Then the relation $\rho_{x}$, as defined by equation (3.9), is a congruence on $S$ if and only if the following condition is satisfied: 
(C) For each $c$ in S and for each y in $Y$, there exists $u$ in $Y$ such that $x^{[-1]} K y$ $=(x c)^{[-1]} K u$.

Proof. By Remark 3.11, $\rho_{x}$ is a right congruence on $S$. Thus to prove the theorem it suffices to show that condition (C) is necessary and sufficient for $\rho_{x}$ to be left compatible. Assume that $\rho_{x}$ is a left congruence on $S$ and let $s, t \in[y]$ where for convenience $x^{[-1]} K y$ is denoted by $[y]$. Thus $x s=x y=$ $x t$ and therefore $(s, t) \in \rho_{x}$. For any $c$ in $S, \rho_{x}$ being left compatible implies that $(c s, c t) \in \rho_{x}$. Hence there exists $y^{\prime}$ in $Y$ such that $c s, c t \in\left[y^{\prime}\right]$, that is, $x c s$ $=x c t=x y^{\prime}$. Thus $s, t \in(x c)^{[-1]} K y^{\prime}$.

Conversely, let $(s, t) \in \rho_{x}, c \in S$, and assume condition (C) holds. Since $(s, t) \in \rho_{x}$, there exists $y$ in $Y$ such that $x s=x t=x y$. By hypothesis then, there exists $y^{\prime}$ in $Y$ such that $x c s=x c t=x y^{\prime}$. This last equation implies that $c s, c t \in\left[y^{\prime}\right]$, that is, $(c s, c t) \in \rho_{x}$.

THEOREM 3.17. Let $S$ be a semigroup containing minimal right ideals and denote their union by $R$. Then the relation $\rho_{R}$ defined by

$$
\rho_{R}=\{(a, b) \in S \times S: r a=r b \text { for all } r \text { in } R\}
$$

is a congruence on $S$. Moreover, the following are equivalent:

(1) $\rho_{x}$ is a congruence for some $x$ in $R$.

(2) $\rho_{x}$ is a congruence for every $x$ in $R$.

(3) $\rho_{x}=\rho_{R}$ for some $x$ in $R$.

(4) $\rho_{x}=\rho_{R}$ for every $x$ in $R$.

(5) $\rho_{x}=\rho_{y}$ for all $x, y$ in $R$.

(6) $\rho_{R}$ is the minimum right group congruence on $S$.

Proof. By Remark 3.11, $\rho_{R}$ is a right congruence on $S$ with $X=R$. To show that $\rho_{R}$ is left compatible, let $(a, b) \in \rho_{R}$ and $c \in S$. For any $r \in R, r c$ is in $R$. Hence $r c a=r c b$, and therefore $(c a, c b) \in \rho_{r}$ for all $r$ in $R$.

(1) implies (3). Assume that for some $x \in R, \rho_{x}$ is a congruence on $S$. For $r \in R$ there exists $u \in S$ such that $r x u=r$ since $r x S$ is the MRI of $S$ containing $r$. If $(a, b) \in \rho_{x}$, then $(u a, u b) \in \rho_{x}$ and therefore $x u a=x u b$. Multiplication by $r$ yields $r x u a=r x u b$, i.e., $r a=r b$. Hence $(a, b) \in \rho_{r}$ and therefore $\rho_{x} \subseteq \rho_{r}$ for all $r$ in $R$. Thus $\rho_{R}=\rho_{x}$.

(3) implies (6). There exists $u \in S$ such that $x u=x$ and therefore $x u^{2}$ $=x u$. Thus $\left(u^{2}, u\right) \in \rho_{R}$ and $E_{S / \rho_{R}}$ is nonempty. By part (iv) of Theorem 3.6, $\rho_{x}$ is right simple and hence $S / \rho_{R}$ is a right group by Lemma 2.2 (2).

Next let $\sigma$ be any RGC on $S$. By Theorem 2.7, $\sigma$ ' maps $x S$ onto $S / \sigma$ and 
hence there exists $e \in x S$ such that $e \sigma \xi=e \sigma \in E_{S / \sigma}$, say $e=x s$. If $(a, b)$ $\in \rho_{R}$, then since $\rho_{R}$ is a congruence and equal to $\rho_{x},(s a, s b) \in \rho_{x}$. Hence $x s a=x s b$ and applying $\sigma$ म yields $(x s a) \sigma=(x s b) \sigma$, that is, $(e a) \sigma(e b)$. But by Lemma 2.1, e $\sigma$ is a left identity for $S / \sigma$ and therefore $a \sigma b$. Hence $\rho_{R} \subseteq \sigma$ so $\rho_{R}$ is the MRGC on $S$.

(6) implies (5). Let $(a, b) \in \rho_{k}$ for some $k$ in $R$. Then $k a=k b$ and therefore $(k a) \rho_{R}=(k b) \rho_{R}$. Since $\rho_{R}$ is a RGC it is left cancellative and therefore $a \rho_{R}=b \rho_{R}$, i.e., $(a, b) \in \rho_{r}$ for all $r$ in $R$.

(5) implies (1). Since $\rho_{x}=\rho_{r}$ for all $x, r$ in $R$, then $\rho_{R}=\rho_{x}$ for some $x$ in $R$. By the first portion of the theorem, $\rho_{x}$ is therefore a congruence on $S$.

(5) implies (2). Since $\rho_{R}$ is a congruence and $\rho_{x}=\rho_{y}$ for all $x, y$ in $R$, then $\rho_{R}=\rho_{x}$ for each $x$ in $R$, i.e., $\rho_{x}$ is a congruence for each $x$ in $R$.

It is evident that (2) implies (4) and that (4) implies (5).

COROLLARY 3.18. If $S$ is a commutative semigroup with kernel $R$, then for any $x$ in $R, \rho_{x}$ is the minimum group congruence on $S$ and $S / \rho_{x}$ is isomorphic to $R$.

Proof. For every $x$ in $S, \rho_{x}$ is a right congruence on $S$ and thus a congruence on $S$. In particular, taking $x$ in $R$, Theorem 3.17 and its leftright dual imply that $\rho=\rho_{x}$ is the minimum right simple and left simple congruence on $S$, i.e., $\rho$ is the MGC on $S$.

The kernel $R$ of $S$ is also a group and if $x$ denotes the identity of $R$, then $x r=x t$ for $r, t$ in $R$ if and only if $r=t$. Thus $\rho^{\natural}$ is one-to-one on $R$, so, by Theorem 2.7 ,

$$
S / \rho_{x}=S \rho_{x}^{\natural}=R \rho_{x}^{\natural} \cong R \text {. }
$$

REMARK. The converse to the first conclusion of Theorem 3.17 is false. For example, let $S(\cdot)$ be the semigroup defined by the following table:

\begin{tabular}{l|llll}
$\cdot$ & $c$ & $d$ & $e$ & $f$ \\
\hline$c$ & $c$ & $d$ & $e$ & $f$ \\
$d$ & $d$ & $d$ & $d$ & $f$ \\
$e$ & $e$ & $d$ & $d$ & $f$ \\
$f$ & $f$ & $d$ & $d$ & $f$
\end{tabular}

The unique MRI of $S$ is $d S=f S=\{d, f\}$, so $R=\{d, f\}$. Neither $\rho_{f}$ nor $\rho_{d}$ are congruences on $S$. However, $\rho_{R}$ is a congruence, but not a right simple congruence on $S$. 
Another condition which is sufficient to insure the left compatibility of $\rho_{x}$ is given by the following theorem.

THEOREM 3.19. Let $S$ be a semigroup and assume there exists an $x$ in $S$ and $a$ subset $A$ of $S$ such that $A x=x S$. Then $\rho_{x}$ is a congruence on $S$, and moreover, if $A$ is a subsemigroup of $S$, then $S / \rho_{x}$ is a homomorphic image of A.

Proof. Since $\rho_{x}$ is a right congruence on $S$, it suffices to show that $\rho_{x}$ is left compatible. Let $c \in S$ and suppose $a \rho_{x} b$, i.e., $x a=x b$. There exists $y$ in $A$ such that $y x=x c$ and therefore $x c a=y x a=y x b=x c b$, that is, $(c a) \rho_{x}(c b)$.

Next, suppose that $A$ is a subsemigroup of $S$ and define the mapping $\theta$ from $A$ to $S / \rho_{x}$ by

$$
\theta: a \rightarrow c \rho_{x}, \text { for all } a \text { in } A,
$$

where $c$ is an element of $S$ such that $a x=x c$. If $a \in A$ and $a x=x c, a x=$ $x d$, where $c, d \in S$, then $x c=x d$, i.e., $c \rho_{x}=d \rho_{x}$ and $\theta$ is well defined.

If $a, b \in A$ then $a b \in A$, and hence there exists an $r$ in $S$ such that $(a b) x$ $=x r$. Corresponding to $a$ and $b$ there exist $s, t$ in $S$ such that $a x=x s$ and $b x$ $=x t$. But then:

and therefore $r \rho_{x}=(s t) \rho_{x}$. Hence:

$$
x r=(a b) x=a(x t)=x(s t),
$$

$$
(a b) \theta=r \rho_{x}=(s t) \rho_{x}=\left(s \rho_{x}\right)\left(t \rho_{x}\right)=(a \theta)(b \theta),
$$

i.e., $\theta$ is a homomorphism of $A$ into $S / \rho_{x}$.

Lastly, for any $s$ in $S$, there exists $a$ in $A$ such that $a x=x s$ and therefore $\theta$ is a homomorphism of $A$ onto $S / \rho_{x}$.

THEOREM 3.20. Let $S$ be a semigroup and let $I$ be a right ideal of $S$ which contains a right identity $x$. Then the relation $\rho_{x}$ defined by equation (3.9) is a congruence on $S$. Moreover the following are equivalent:

(1) I is a minimal right ideal of $S$.

(2) I is a group.

If this is the case then, in addition,

(3) $\rho_{x}$ is the minimum right group congruence on $S$.

(4) $\rho_{x}$ is the minimum group congruence on $S$.

(5) $\rho_{x}=\rho_{y}$ for all $y$ in $R$, where $R$ is the union of all the minimal right ideals of $S$.

(6) $S / \rho_{x}$ is isomorphic to $I$.

Proof. (1) implies (2). If $I$ is a MRI of $S$, then $I$ is a right group. If $e \in$ 
$E_{I}$ then, by [1, Lemma 1.26, p. 37], $e$ is a left identity for $I$. In particular, $e x$ $=x$. On the other hand, $e x=e$ since $x$ is a right identity for $I$. Hence $\left|E_{x}\right|$ $=1$ and therefore, by [1, Theorem 1.27, p. 38], $I$ is a group.

(2) implies (1). For all $i$ in $I, i I=I$. Thus $I$ is a MRI of $S$.

Now assume that $I$ is a MRI of $S$.

Proof of (3). By (2), $I$ is a group and hence also a right group. Therefore, by Theorem 2.7, $S / \rho_{x}$ is a homomorph of $I$, i.e., $\rho_{x}$ is a RGC on $S$. If $(a, b) \in \rho_{x}$, then $x a=x b$, so $(x a) \sigma=(x b) \sigma$ for any right congruence $\sigma$ on $S$. But $x \sigma$ is then a left identity for $S / \sigma$ and therefore $a \sigma=b \sigma$, i.e., $(a, b)$ $\in \sigma$. Thus $\rho_{x} \subseteq \sigma$.

(4). Combining the proof of (3) with condition (2), we conclude that $\rho_{x}$ is the MGC on $S$.

(5). Since $\rho_{x}$ is a congruence on $S$, it follows from Theorem 3.17 that $\rho_{x}=\rho_{y}$ for all $y$ in $R$.

(6). By (1), $x S=I=I x$ and hence by Theorem 3.19, $S / \rho_{x}^{\natural}$ is a homomorph of $I$. If $(i, j) \in \rho_{x}$ and $i, j \in I$, then $i=i x=j x=j$, i.e., $\rho_{x}$ is one-to-one on $I$.

If $a$ is an element of a regular semigroup $S$, then the set of all inverses of $a$ is denoted by $V(a)$.

CoROllary 3.21. If $S$ is a left group, say $S=G \times L$, where $G$ is a group and $L$ is a left zero semigroup, then for any $x \in S, \rho_{x}$ is the minimum group congruence on $S$ and $S / \rho_{x}$ is isomorphic to $G$.

Proof. For any $a$ in $S, a S$ is a right ideal of $S$ containing $a$. If $b \in a S$, then $b=a t$ for some $t$ in $S$. By the dual of Lemma 2.2, $S$ is regular and thus $b t^{\prime}=a t t^{\prime}$ for every $t^{\prime}$ in $V(t)$. Since $t t^{\prime}$ is idempotent, by the dual of Lemma $2.1, t t^{\prime}$ is a right identity for $S$, so $b t^{\prime}=a$. Thus $b S=a S$, so $a S$ is a MRI of $S$. Therefore each element of $S$ is contained in some MRI of $S$, i.e., $S=R$, where $R$ denotes the union of all MRI's of $S$.

If $e$ is an idempotent of $S$ then by the above paragraph, $e S$ is a MRI of $S$, and $e$ is a right identity since $(e S) e=e S$. By Theorem 3.20,eS is a group, $\rho_{x}=\rho_{R}\left(=\rho_{S}\right)$ is the MRGC on $S$, and $S / \rho_{x} \cong e S$. By Lemma 2.2 (4), $e S \cong G$.

A homogroup is a semigroup $S$ containing an ideal $A$ which is a subgroup of $S ; A$ is sometimes called a group ideal of $S$.

COROLlaRY 3.22. Let $S$ be a homogroup, with group ideal I. For every $x$ in $I, \rho_{x}$ is the minimum group congruence on $S$ and $S / \rho_{x}$ is isomorphic to $I$. 
THEOREM 3.23. Let $S$ be a semigroup which contains a right zero. Denote the set of all right zeros of $S$ by $R$, and define $\rho_{R}$ as in equation (3.10). Then:

(1) $R$ is the (unique) minimal right ideal of $S$.

(2) Every right simple homomorph of $S$ is a right zero semigroup.

(3) $\rho_{R}$ is a congruence on $S$.

(4) If $\rho_{R}$ is right simple then it is the minimum right group congruence on $S$, and $S / \rho_{R}$ is isomorphic to $R$.

Proof. The proof of (1) is evident. For (2), let $\sigma$ be any right simple congruence on $S$. By Theorem 2.7, the induced homomorphism $\sigma^{\natural}$ maps $R$ onto $S / \sigma$ and therefore $\sigma$ is a RZC.

(3) By Remark 3.11, it suffices to show that $\rho_{R}$ is left compatible. For any $c$ in $S$ and $r$ in $R, r c \in R$. Thus if $a \rho_{R} b$, then $(r c) a=(r c) b$ for all $c$ in $S$, $r$ in $R$. Hence $(c a, c b) \in \rho_{r}$ for all $r$ in $R$ so $(c a, c b) \in \rho_{R}$.

(4) If $\rho_{R}$ is right simple, then since $r^{2} \rho_{R} r$ for all $r$ in $R, \rho_{r}$ is a RGC. If $\sigma$ is any RGC on $S$ and $(a, b) \in \rho_{R}$, then $r a=r b$ for $r$ in $R$ implies that ( $r a$, $r b) \in \sigma$. But $r^{2}=r$ implies that $r \sigma \in E_{S / \sigma}$ and therefore $r \sigma$ is a left identity for $S / \sigma$. Thus $a \sigma b$ and $\rho_{R} \subseteq \sigma$.

Lastly, if $(s, t) \in \rho_{R}$ and $s, t \in R$ then $s=r s=r t=t$ for all $r$ in $R$. Thus $\rho_{R}^{\natural}$ is one-to-one on $R$ and $\rho_{R}^{\natural}$ maps $R$ isomorphically onto $S / \rho_{R}$.

For comparison, recall that in Corollary $2.8, S$ having a left zero implied that the MRGC on $S$ was $\omega$.

The next theorem is closely related to Theorem 3.17 in that $\rho_{R}$ is assumed to be the MRGC on $S$ and the structure of $S$ is then determined.

THEOREM 3.24. Let $S$ be a semigroup containing minimal right ideals and denote the union of the minimal right ideals of $S$ by $R$. Suppose the relation $\rho_{R}$ on $S$ defined by

$$
\rho_{R}=\{(a, b) \in S \times S: r a=r b \text { for all } r \in R\}
$$

is the minimum right group congruence on $S$. Then:

(1) $E_{S} \neq \square$.

(2) Every minimal right ideal of $S$ is isomorphic to $S / \rho_{R}$.

(3) Each equivalence class $A$ of $\rho_{R}$ contains a unique minimal right neat subset of $S$, that is, $A$ contains exactly one element of each minimal right ideal of $S$.

(4) Let $B$ be an index set for the collection of minimal right neat subsets of $S$ specified in (3). Then $\rho_{R}$ can be characterized as follows:

$$
\rho_{R}=\left\{(a, b) \in S \times S: K_{i} a=K_{i} \text { b for each } i \text { in } B\right\} \text {. }
$$

Proof. For convenience, $\rho_{R}$ will be denoted by $\rho$ in this proof. 
(1) Let $e \rho \in E_{S / \rho}$. Since $S / \rho$ is right simple, $e \rho$ is a right neat subset of $S$ and hence contains a MRN subset of $S$ meeting each MRI of $S$. Let $x$ $\in I \cap e \rho$ for some MRI, $I$, of $S$. Since $x \in e \rho$, then $x x=x e$ and therefore $x e x=x^{3}$. But $e \rho$ is a left identity for $S / \rho$ and hence $(e x) \rho x$, which implies $x e x=x^{2}$. Combining this with $x e x=x^{3}$ yields $x^{2}=x^{3}$. Since $x^{2} I=I$, then to each $x$ in $I \cap e \rho$ there corresponds $u$ in $I$ such that $x^{2} u=x$. Thus

$$
x=x^{2} u=x^{3} u=x\left(x^{2} u\right)=x^{2} \text {. }
$$

So $x \in E_{S}$ and in particular, $x$ is a left identity for $I$.

(2) Let $I$ be a MRI of $S$ and let $x$ be a left identity element of $I$. For any $i$ in $I, i I=I$ and hence, since $E_{I} \neq \square, I$ is a right group. By Theorem 2.7, $I$ is mapped onto $S / \rho$ by the natural homomorphism $\rho^{\natural}$. If $a, b \in I$ and $a \rho b$, then $x a=x b$. But $x$ is a left identity for $I$ and therefore $\rho$ is the identity relation on $I$, i.e., $I \cong S / \rho$.

(3) Let $a \rho$ be any $\rho$-class of $S$ and suppose that $i, j \in I \cap a \rho$, where $I$ and $x$ are as defined in (2). Since $\rho=\rho_{x}$, then $x i=x j=x a$. Since $x$ is a left identity for $I, i=j=x a$, that is, $|I \cap a \rho|=1$. Hence $a \rho$ contains exactly one cross-section of the MRI's of $S$.

(4) If $(a, b)^{\circ} \in \rho$ then $x a=x b$ for all $x$ in $R$. Hence for each $i$ in $B$ and for each $x$ in $K_{i}, x a=x b$, i.e., $K_{i} a=K_{i} b$.

Conversely, if $a, b \in S$ are such that $K_{i} a=K_{i} b$ for each $i$ in $B$, then there exist $h, k$ in $K_{i}$ such that $h a=k b$ and therefore, by Theorem 3.2 (iii), $h=k$. Thus $h a=h b$ for each $i$ in $B$ and for each $h$ in $K_{i}$. By (3), $R=$ $\cup\left\{K_{i}: i\right.$ in $\left.B\right\}$. Thus $h a=h b$ for each $h$ in $R$, i.e., $(a, b) \in \rho$.

The following result is a partial converse to Theorem 3.24.

THEOREM 3.25. Lèt $S$ be a semigroup containing minimal right ideals. Assume that for some minimal right neat subset $K$ of $S$, and for some $x$ in $K$, $\rho_{x}$ satisfies the following two conditions.

(1) Each $\rho_{x}$-class of $S$ contains a unique minimal right neat subset of $S$.

(2) If $(a, b) \in \rho_{x}$, then $H a=H b$, where $H$ is any of the unique minimal right neat subsets from condition (1).

Then $\rho_{x}$ is the minimum right group congruence on $S$.

Proof. Let $C$ be the set of MRN subsets of $S$ provided by hypothesis (1). Index $C$ by a set $B$, say $C=\left\{K_{i}: i \in B\right\}$. Thus $B$ is also an index set for the $\rho_{x}$-classes of $S$. We first show that:

$$
R=\cup\left\{K_{i}: i \in B\right\}
$$

where $R$ denotes the union of all MRI's of $S$. Clearly the right side of (3.26) 
is contained in the left. Conversely, if $r \in R$, then $r$ lies in some MRN subset of $S$.

Since $\rho_{x}$ is a right congruence on $S$, it remains to show that $\rho_{x}$ is left compatible. By (3.26), $x$ in $K$ implies that $x \in K_{i}$ for some $i$ in $B$. Let $(a, b)$ $\in \rho_{x}$ and $c \in S$. The set $K_{i} c$ is a MRN subset by Theorem 3.2 (iv), and hence, by hypothesis (2), $K_{i} c a=K_{i} c b$. But then by Theorem 3.2 (iii), $x c a$ $=x c b$ and therefore $\rho_{x}$ is a congruence. Moreover, by Theorem $3.17(6), \rho_{x}$ is the MRGC on $S$.

At this point it would seem natural to ask if the existence of MRN subsets is a necessary and sufficient condition for a minimum right simple congruence to exist. The necessity of this condition is an open question, whereas this condition is not sufficient. Consider, for example, the full transformation semigroup $T_{X}$ on the set $X=\{1,2\}$. The constant transformations of $X$ form a MRI of $T_{X}$, say $R$, but the only right simple congruences on $T_{X}$ are $\iota$ and $\omega$. In fact $\rho_{R}=\iota$.

\section{REFERENCES}

1. A. H. Clifford and G. B. Preston, The Algebraic Theory of Semigroups, Vol. I, Mathematical Surveys (No. 7), Amer. Math. Soc., Providence, Rhode Island, 1961.

2. $\longrightarrow$ The Algebraic Theory of Semigroups, Vol. II, Mathematical Surveys (No. 7), Amer. Math. Soc., Providence, Rhode Island, 1967.

3. P. Dubreil, Quelques Problemes d'Algebra lies a la theorie des demi-groupes, Colloque d'Algebre superieure, Bruxelles (1956), 29-44.

4. Jean-Claude Fontaine, Sur certaines equivalences definies dans un demigroupe et liees a l'equivalence reversible generalisee, C. R. Acad. Sci. Paris, (Ser. A-B) V264 (1967), 781-784. 5. P. Lefebvre, Demi-groupes admettant des complexes nets a droite minimax, Comptes Rendus Acad. Sci. Paris, V247 (1958), 393-396.

6. - Sur les groupes homomorphes a un demi-groupe; demi-groupes admettant un groupe homomorphe maximum, C. R. Acad. Sci. Paris, V248 (1959), 2277-2279.

Received October 13, 1972 and in revised form June 6, 1974. The results of this paper were included in the author's doctoral dissertation, directed by Professor Donald W. Miller of the University of Nebraska.

Glassboro State College 


\section{PACIFIC JOURNAL OF MATHEMATICS}

\section{EDITORS}

RICHARD ARENS (Managing Editor)

University of California

Los Angeles, California, 90024

\section{R. A. BEAUMONT}

University of Washington

Seattle, Washington 98105

\section{J. DugunDJI}

Department of Mathematics University of Southern California Los Angeles, California 90007

D. Gillbarg AND J. MilgRAM

Stanford University Stanford, California 94305

\section{ASSOCIATE EDITORS}

E. F. BECKENBACH

B. H. NEUMANN

SUPPORTING

UNIVERSITY OF BRITISH COLUMBIA

UNIVERSITY OF CALIFORNIA

MONTANA STATE UNIVERSITY

UNIVERSITY OF NEVADA

NEW MEXICO STATE UNIVERSITY

OREGON STATE UNIVERSITY

UNIVERSITY OF OREGON OSAKA UNIVERSITY
F. WOLF

K. YosHIDA

INSTITUTIONS

UNIVERSITY OF SOUTHERN CALIFORNIA

STANFORD UNIVERSITY

UNIVERSITY OF TOKYO

UNIVERSITY OF UTAH

WASHINGTON STATE UNIVERSITY

UNIVERSITY OF WASHINGTON

AMERICAN MATHEMATICAL SOCIETY

NAVAL WEAPONS CENTER

The Supporting Institutions listed above contribute to the cost of publication of this Journal, but they are not owners or publishers and have no responsibility for its content or policies.

Mathematical papers intended for publication in the Pacific Journal of Mathematics should be in typed form or offset-reproduced, (not dittoed), double spaced with large margins. Underline Greek letters in red, German in green, and script in blue. The first paragraph or two must be capable of being used separately as a synopsis of the entire paper. Items of the bibliography should not be cited there unless absolutely necessary, in which case they must be identified by author and Journal, rather than by item number. Manuscripts, in duplicate if possible, may be sent to any one of the five editors. Please classify according to the scheme of Math. Rev. Index to Vol. ${ }^{39}$. All other communications to the editors should be addressed to the managing editor, or Elaine Barth, University of California, Los Angeles, California, 90024.

100 reprints are provided free for each article, only if page charges have been substantially paid. Additional copies may be obtained at cost in multiples of 50 .

The Pacific Journal of Mathematics is issued monthly as of January 1966. Regular subscription rate: $\$ 72.00$ a year (6 Vols., 12 issues). Special rate: $\$ 36.00$ a year to individual members of supporting institutions.

Subscriptions, orders for back numbers, and changes of address should be sent to Pacific Journal of Mathematics, 103 Highland Boulevard, Berkeley, California 90708.

\section{PUBLISHED BY PACIFIC JOURNAL OF MATHEMATICS, A NON-PROFIT CORPORATION}

Copyright (C) 1974 by Pacific Journal of Mathematics

Manufactured and first issued in the U.S.A. 


\section{Pacific Journal of Mathematics}

\section{Vol. 54, No. $2 \quad$ June, 1974}

John Edward Coury, Walsh series with coefficients tending monotonically to

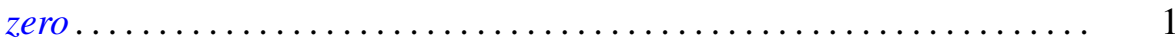

Patrick Michael Fitzpatrick and Walter Volodymyr Petryshyn, Fixed point theorems for multivalued noncompact acyclic mappings ............

Irving Leonard Glicksberg, More on Phragmén-Lindelöf for function

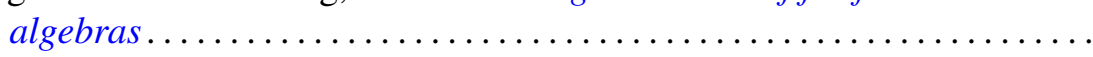

Adilson Goncalves, Structural constants. II .................. 39

Richard P. Gosselin, Closure theorems for affine transformation groups .... 53

Ralph Peter Grimaldi, Baer and UT-modules over domains ........... 59

Edward Grossman, On the prime ideal divisors of $\left(a^{n}-b^{n}\right) \ldots \ldots \ldots \ldots . \ldots 73$

A. Hedayat and Ester Seiden, On the theory and application of sum composition of Latin squares and orthogonal Latin squares.......... .

Gerald L. Itzkowitz, Continuous measures, Baire category, and uniform continuity in topological groups ......................... 115

Francis Masat, Right simple congruences on a semigroup ............ 127

Robert Harvey Oehmke, Right congruences and semisimplicity for Rees matrix semigroups..................................

Qazi Ibadur Rahman and Jan Stankiewicz, Differential inequalities and local valency . . . . . . . . . . . . . . . . . . . . . . . . . . . . . . . . . . . . . 165

William John Reed, Random points in a simplex ................ 183

Mohan S. Shrikhande, Strongly regular graphs and group divisible

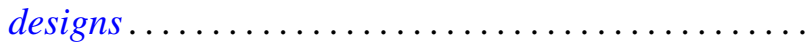

Zahava Shmuely, The structure of Galois connections ... . .

Robert C. Shock, Dual generalizations of the Artinian and Noetherian

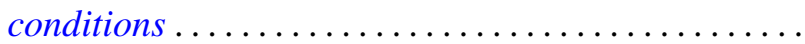

Arne Stray, Approximation and interpolation for some spaces of analytic functions in the unit disc ........................

Eldon Jon Vought, Monotone decompositions into trees of Hausdorff continua irreducible about a finite subset ............

James Wirth, The mapping cylinder axiom for WCHP fibrations ... 263

Gordon S. Woodward, Invariant means and ergodic sets in Fourier analysis... 